\title{
Fitness in Evolutionary Biology
}

\author{
By Thomas F. Hansen \\ University of Oslo, Department of Biology, CEES \& Evogene, PB 1066, 0316 Oslo, \\ Norway. Email: thomas.hansen@ bio.uio.no
}

\begin{abstract}
A review of the concept of "fitness" as it is used in evolutionary theory.
\end{abstract}

Key words: Fitness, Natural selection 


\section{Note of publication}

This manuscript owes its odd format to the fact that it was originally commissioned and prepared as an entry on the topic "Fitness" for the Oxford Bibliographies in Evolutionary Biology. The Oxford Bibliographies neglected to inform me that they were unable to publish mathematical equations, and for this reason the accepted entry was withdrawn by mutual agreement between the editor and me. I place it on the preprint server in the hope that it could be of use.

\section{Table of contents}

Introduction

Overview

Fitness in the Theory of Natural Selection

Fitness and the Calculus of Natural Selection

The Fundamental Theorem of Natural Selection

Fitness in Evolutionary Explanation

Malthusian Fitness

Measuring Fitness I: Fitness Components

Measuring Fitness II: Scaling and Transformation

Fitness in Varying Environments

Fitness in Structured Populations

Fitness and Levels of Selection

Inclusive Fitness

Fitness in Finite Populations

Fitness in the Philosophy of Biology

Alternative Characterizations of Fitness

Acknowledgements 


\section{Introduction}

Fitness is a key concept in evolutionary biology embedded at the core of the theory of natural selection. It may be tentatively defined as the ability to survive and reproduce. The term itself started life with Herbert Spencer's rather vague metaphor "survival of the fittest", but during the modern synthesis it gradually acquired a precise meaning in formal mathematical descriptions of selection. Here fitness is a measure of the change in the numbers of a type over an episode of selection. Assigning fitness to traits or types is a basic element of evolutionary explanation. If a trait causes a change in fitness it will be affected by selection, and quantifying the link to fitness provides the means for predicting of how strong this effect will be. Using the standard textbook example of evolution of melanic moths in areas with industrial pollution, we may have found that dark-colored (melanic) moths have, say, a $1 \%$ probability of being picked off by birds while resting on branches, while light-colored moths, which are more conspicuous on branches void of the lichens they were adapted to hide among, have a probability of, say, $2 \%$ of being taken by birds. Assigning survival fitnesses of $99 \%$ and $98 \%$ to the two types, we can calculate the change in frequency of the two types due to selection by birds. Combined with information about inheritance and other evolutionary forces this can be used to predict or explain the evolution of the traits. In this way, selection explanations are fundamentally based on relating measurements of fitness to measurements of traits, and a large body of mathematical, statistical and experimental methods has been developed to this end. Due to its central role in evolutionary explanation and the many nuances of its application, the fitness concept has drawn attention among theoreticians and philosophers of biology. There is a large literature concerned with formal characterizations of fitness and solving the semantic problems they give rise to. There are also many alternative conceptions of fitness with varying degrees of connection to the actual use of the concept in evolutionary research.

\section{Overview}

There was no mention of "fitness" in Darwin's or Wallace's original descriptions of natural selection. The term was introduced by Spencer (1864) through the "survival of the fittest" metaphor. This metaphor was accepted by both Darwin and Wallace as a good description of natural selection. In the 1920s and 30s Fisher, Haldane, Wright and others produced a series of papers that collectively established a mathematical description of natural selection and other evolutionary forces based on the gene concept. In this work alleles and genotypes were assigned numbers corresponding to fitness under a variety of names and descriptions such as selection coefficients, selective values, etc. Fisher (1930) established the use of "fitness" as a general term for this number, and linked it to the Malthusian rate of population growth for the genotype in question (see also Fisher 1922). This was used in his fundamental theorem of natural selection stating that the increase in (mean) fitness due to selection is proportional to the variance in fitness. The fundamental theorem implies that selection always increases mean fitness and has served as a justification for fitness optimization as a research strategy in evolutionary biology. Fitness optimization is reflected in concepts such as the adaptive landscape (Wright 1932) or fitness landscape, which plots fitness against traits, genotypes or genotype frequencies, and depicts evolution as an uphill walk in the topography ending on local fitness peaks (Frank 2012a). Today, much evolutionary research is based on studying the 
relationship of traits to fitness. This requires measurement of fitness, which usually takes the form of identifying some component of fitness that carries the causal connection between trait and selection. Theoretical research has addressed the measurement of fitness under various complications involving population structure, frequency dependence, levels of selection, kin selection, finite population size, multigenerational effects, etc. This has also given rise to a multitude of different definitions and conceptualizations of fitness aimed at solving problems or capturing its use in different contexts. De Jong (1994), Metz (2008), Barker (2009), Abrams (2012) are general reviews describing and classifying different conceptions of fitness. An overview of uses of fitness in evolutionary explanation can be found in standard textbooks in evolutionary biology such as Futuyma (2013).

Abrams, M. 2012. Measured, modeled, and causal conceptions of fitness. Frontiers in Genetics 3: 196.

Review of different notions of fitness by a philosopher. Develops a terminology distinguishing Mathematical, Statistical, Parametric and Token fitness. Argues that parametric fitness, defined as an underlying property of a type that is estimated by statistical fitness, is what biologists have in mind as the causal component of natural selection. Token fitness is the realized fitness of an individual.

Barker, J. S. F. 2009. Defining fitness in natural and domesticated populations. In J. Van der Werf, H.-U. Graser, R. Frankham and C. Gondro (Eds.). Evolutionary and breeding perspectives on genetic resource management., Springer, Heidelberg, Pp. 3-14.

General review of the history of fitness and a classification of the different fitness concepts.

De Jong, G. 1994. The fitness of fitness concepts and the description of natural selection. Quart. Rev. Biol. 69: 3-29.

General review of different notions of fitness from a population-genetics perspective.

Fisher, R. A. 1922. On the dominance ratio. Proc. R. Soc. Edinburgh 42: 321-341.

This paper may contain the first mention of fitness in the modern sense of change in numbers of types, but the term was not generally used by neither Fisher, Haldane or Wright before the publication of Fisher's (1930) book.

Fisher, R. A. 1930. The genetical theory of natural selection. Oxford University press [the varioum edition].

This book established the modern population genetics use of fitness. Links fitness to the Malthusian growth rate of an allele, and presents the fundamental theorem of natural selection. A second edition appeared in 1958.

Frank, S. A. 2012a. Wright's adaptive landscape versus Fisher's fundamental theorem. In Svensson, E. and R. Calsbeek (Eds.). The adaptive landscape in evolutionary biology. Oxford University press.: Pp 41-57. 
A clear discussion of the debates between Fisher and Wright with explanations of how they understood the fundamental theorem and the adaptive landscape. Argues that Fisher intended the fundamental theorem as a fundamental law about natural selection and that Wright, like most later commentators, misunderstood it as a dynamical model of evolution by natural selection. Discusses the definition of fitness.

Futuyma, D. J. 2013. Evolution. Third Edition. Sinauer. Sunderland, MA.

High-end textbook of evolutionary biology with much material on the use of fitness and its role in the general theory. Gives several characterizations of fitness such as "The fitness .. of a biological entity is its average per capita rate of increase in numbers" (p285), and "The fitness of a genotype is the average per capita lifetime contribution of individuals of that genotype to the population after one or more generations" (p312).

Metz, J. A. J. 2008. Fitness. In S. E. Jørgensen and B. D. Fath (Eds.). Encyclopedia of Ecology, vol 2., Elsevier, Oxford. Pp. 1599-1612.

General review of fitness from an adaptive-dynamics perspective.

Spencer, H. 1864. The Principles of Biology, Vol. 1, Williams and Norgate, London \& Edinburgh.

First appearance of the term "fitness" in the context of natural selection, and of the characterization of natural selection as "survival of the fittest". Darwin adopted this in the 5th edition of "The Origin of Species", although he did use terms such as "fit" and "fitted" in earlier editions.

Wright, S. 1932. The roles of mutation, inbreeding, crossbreeding and selection in evolution. Proc. Sixth Int. Con. Genetics 1: 356-366.

Introduces the adaptive-landscape metaphor for evolution by natural selection as a hill-climbing process in a fitness landscape.

\section{Fitness in the Theory of Natural Selection}

Stearns (1976) defined fitness as something everyone understands but no one can define precisely and Williams (1970) regarded fitness as a primitive term not definable within the theory of natural selection itself. These sentiments reflect the fact that fitness is a concept that is thoroughly embedded in the theory of natural selection, and although its role in this theory is usually precise and easy to understand, it is hard to capture in verbal definitions. Loosely based on Lewontin (1970), the conditions for evolution by natural selection can be summarized as follows:

1. Individuals in a population have different properties (there is variation).

2. The properties of the individuals affect their ability to survive and reproduce.

3. The properties of the individuals are heritable (offspring are more similar to their parents than to other individuals in the population). 
If conditions 1 and 2 are fulfilled, we have natural selection, which ceteris paribus changes the statistical distribution of properties in the population, and if condition 3 about heritability is also fulfilled at least some of this change will be transferred to the next generation and we have evolution by natural selection. In evolutionary theory fitness has become the shorthand for "ability to survive and reproduce". From this we can identify some of its key properties. The first is that fitness is a dispositional concept referring to an ability or propensity for survival and reproduction, and not to the actual realization of such. The second is that fitness needs to be linked with particular properties (e.g. traits, genotypes) to serve an explanatory role. Fitness is therefore assigned to categories or types, sets of individuals with a common property. One may talk about or measure the fitness of an individual organism as the fitness realized or predicted by its properties, but this has no formal role in the theory. The third observation is that selection and fitness are logically independent from inheritance (transmission of properties). Fitness applies to an episode of selection and how the changes caused by this episode are transmitted across generations is a different and more complicated matter. Some general expositions of evolution by natural selection from different perspectives can be found in Sober $(1984,2011)$, Williams (1992), Bell (1997), Okasha (2006), and Godfrey-Smith (2009).

Bell, G. 1997. Selection: the mechanism of evolution Chapman \& Hall.

A comprehensive account of selection as a process. Also in a second edition.

\section{Godfrey-Smith, P. 2009. Darwinian populations and natural selection. Oxford University Press.}

A recent discussion of the philosophy of natural selection.

Lewontin, R. C. 1970. The units of selection. Ann. Rev. Ecol. Syst. 1: 1-18.

One of the first bare-bones statements of the essential criteria for evolution by natural selection to occur. His description is similar to the three criteria above except that he requires that fitness differences and not trait differences are heritable (see Okasha 2006 for criticism of this). Points out that this definition of natural selection may apply to entities at many levels such as alleles, individuals, groups and species.

Okasha, S. 2006. Evolution and the levels of selection, Oxford University Press.

A readable general discourse on natural selection by a philosopher. Gives a clear presentation of the Price theorem. Emphasis is on levels of selection.

Sober, E. 1984a. The nature of selection: Evolutionary theory in philosophical focus. Bradford books.

An influential and exceptionally well-written introduction to the philosophy of natural selection. Distinguishes selection for a trait vs. selection of a trait, where the former means that the trait has a causal influence on fitness and the later that the trait is selected because it is correlated with other traits that influence fitness (cmp. direct and 
indirect selection). Also good discussions of causality, levels of selection and the tautology problem.

Sober, E. 2011. A priori causal models of natural selection. Australasian J. Philosophy 89: 571-589.

A more recent perspective from Sober. Argues that the principle of natural selection is an analytic and not an empirical law, but still provides causal explanation.

Stearns, S. C. 1976. Life history tactics: a review of the ideas. Quarterly Review of Biology 51: 3-45.

Review of life-history theory with a much-quoted non-definition of fitness.

Williams, G. C. 1992. Natural Selection: Domains, Levels, and Challenges. Oxford univ. press.

Thought-provoking book on unsolved problems and puzzles in the theory of evolution by natural selection. Extensive discussions of levels of selection.

Williams, M. B. 1970. Deducing the consequences of evolution: A mathematical model. $J$. Theor. Biol. 29: 343-385.

An attempt by a philosopher to axiomatize the theory of natural selection. Argues that fitness should be regarded as a primitive term that can not be defined within the theory itself.

\section{Fitness and the Calculus of Natural Selection}

Consider a population of different types, which are sets of individuals (or other entities) with a particular trait, genotype, or some other common property, and let $N_{i}$ be a measure of number or amount of individuals of type $i$. Then consider an episode of selection that changes the number from $N_{i}$ to $N^{\prime}{ }_{i}$. This change may be due to survival or reproduction. The fitness of type $i$ over this episode of selection, which may be anything from a short event to a generation or more, is then defined as $W_{i}=$ $N^{\prime} i / N_{i}$. This is the absolute fitness of the type. Population geneticists are usually concerned with the changes in frequency of types, and the frequency of type $i$ after selection is

$$
p_{i}^{\prime}=\frac{N_{i}^{\prime}}{\sum_{j} N_{j}^{\prime}}=\frac{W_{i} N_{i}}{\sum_{j} W_{j} N_{j}}=\frac{W_{i}}{W} p_{i}=w_{i} p_{i}
$$

where $p_{i}=N_{i} / N$ is the frequency of type $i, N=\Sigma_{j} N_{j}$ the total population size, and $W=$ $\Sigma_{j} W_{j} p_{j}$ the mean fitness of the population (all before selection). The entity $w_{i}=W_{i} / W$ is the relative fitness of type $i$. Hence, absolute fitnesses describe changes in numbers of types and relative fitnesses describe changes in frequencies of types. This is a description of the effects of selection and not evolution. Types may also change due to transmission effects, such as imperfect inheritance. In population genetics the types under consideration are often alleles, because these replicate with high accuracy so 
that transmission effects can be ignored. The fitness of an allele depends on what other alleles it co-occurs with. For example, in a diploid system with one locus and two alleles, $B$ and $b$, the allele $B$ sometimes occurs as a homozygote, $B B$, and sometimes as a heterozygote $B b$. The fitness of $B$ is then a weighted average of the fitnesses of the two genotypes, $W_{B B}$ and $W_{B b}$. This is called the marginal fitness of $B$, and under random mating it is $W_{B}=W_{B B} p+W_{B b q}$, where $p$ is the frequency of $B$ and $q$ the frequency of $b$. This gives the standard textbook equation for selection on a single locus with two alleles:

$$
p^{\prime}=w_{B} p=\frac{W_{B B} p+W_{B b q}}{W} p
$$

If the episode covers all selection within a generation and there are no transmission effects (e.g. no mutation), the frequency $p^{\prime}$ will also be the frequency of the allele $B$ among zygotes in the next generation (e.g. Crow \& Kimura 1970).

Crow, J. F. and M. Kimura. 1970. An introduction to population genetics theory. Harper \& Row, New York.

Classic text on mathematical population genetics summarizing the standard models of allele-frequency change.

\section{The fundamental Theorem of Natural Selection}

In 1930 Fisher presented his fundamental theorem stating that "the rate of increase in fitness .. is equal to .. genetic variance in fitness". His derivation was obscure and incomplete, and for many decades it was not understood why Fisher claimed it to be a general result when it seemed to be based on a number of specific assumptions. In 1970, George Price derived a general result of the same type as Fisher and gave an interpretation of the fundamental theorem consistent with what Fisher had claimed (Price 1970, 1972a). Price's theorem also remained underappreciated until the 1990s when it was taken up, explained and used by many (e.g. Frank \& Slatkin 1992). Price's theorem states that the change in the mean (expectation, E[]) of a trait, $z$, over an episode of selection can always be expressed as

$$
\Delta \mathrm{E}[z]=\operatorname{Cov}[w(z), z]+\mathrm{E}[w(z) \Delta z]
$$

where $w(z)$ is the relative fitness as a function of trait value $z$. The first covariance term describes the effect of selection and the second term describes the effects of transmission by allowing the possibility that entities with trait value $z$ change or give rise to entities with trait value $z+\Delta z$ over the episode of selection. The theorem follows by simple calculation from the definition of fitness as change in numbers of a type as explained in the previous section. It makes no assumptions about genetic details or mating system. A version of the fundamental theorem follows by replacing the trait $z$ with fitness, $W$, in the selection part of the equation:

$$
\Delta \mathrm{E}[W]=\operatorname{Cov}\left[\frac{W}{W}, W\right]=\frac{\operatorname{Var}[W]}{W} .
$$


Hence, in this sense, the fundamental theorem is completely general as a claim about natural selection, but it is not a claim about evolution by natural selection, as it leaves out the transmission effects. Lucid explanations, derivations and interpretations of the Price theorem can be found in Frank (1995, 2012b), Heywood (2005), Okasha (2006, op. cit.) and Kerr and Godfrey-Smith (2009). Proofs and interpretations of the fundamental theorem in Fisher's sense can be found in e.g. Ewens (1989), Edwards (1994) and Grafen (2015).

Edwards A. W. F. 1994. The fundamental theorem of natural selection. Biological Reviews 69: 443-474.

Comprehensive non-technical review of older work and interpretations of the fundamental theorem, and the debates between Fisher and Wright.

Ewens, W. J. 1989. An interpretation and proof of the fundamental theorem of natural Selection. Theor. Pop. Biol. 36: 167-180.

This paper provides the first detailed proof of the fundamental theorem in the way Fisher may have intended it. In Ewens' interpretation the fundamental theorem describes a partial change across generations that is due to selection. This is less general than the simple interpretation above and also states that the mean change in fitness across generations depends on the additive genetic variance.

Frank, S. A. 1995. George Price's contribution to evolutionary genetics. J. Theor. Biol. 175: 373-388.

A short biography of George Price's strange life with a clear exposition of the Price theorem and its applications.

Frank, S. A. 2012b. Natural selection. IV. The Price equation. J. Evol. Biol. 25: 1002-1019.

Recent review of uses and comments on the Price theorem. Defends it against some criticisms and derives it in various forms.

Frank, S. A. \& M. Slatkin. 1992. Fisher's fundamental theorem of natural selection. TREE 7: 92-95.

One of the first papers explaining the Price interpretation of the fundamental theorem in a nontechnical way with biological examples. The emphasis is on how changes in the environment can be expressed as transmission effects. For example, soft selection when individuals compete against each other may increase mean fitness relative to a constant environment, but the increased competitiveness in the population will also deteriorate the environment and decrease fitness. This later effect may be described as a negative transmission term.

Grafen, A. 2015. Biological fitness and the fundamental theorem of natural selection. Am. Nat. 186: 1-14.

Technical paper claiming to give the first satisfactory proof of the fundamental 
theorem.

Heywood, J. S. 2005. An exact form of the breeder's equation for the evolution of a quantitative trait under natural selection. Evolution 59: 2287-2298.

Presents various further decompositions of the Price equation and discusses its relation to other equations for evolution by natural selection.

Kerr, B. and P. Godfrey-Smith. 2009. Generalization of the Price equation for evolutionary change. Evolution 63: 531-536.

A generalization of the Price equation to describe the mapping between two sets of individuals including, but not limited to, ancestors and descendants.

Price, G. R. 1970. Selection and covariance. Nature 227: 520-521.

First presentation of what has become known as Price's theorem or Price's equation decomposing the response to selection into a covariance term describing the change due to selection and a transmission term.

Price, G. R. 1972a. Fisher's 'fundamental theorem' made clear. Ann. Hum. Genet., Lond. 36: 129-140.

Gives an interpretation of the fundamental theorem as a general result about selection similar to the selection term of the Price equation. Makes some negative remarks about the importance of the theorem and Fisher's presentation of it.

\section{Fitness in Evolutionary Explanation}

A trait will be under selection if it has, or is correlated with, a causal effect on fitness, and measurements of the strength of this effect can be used to make quantitative predictions about how the trait distribution will be changed by selection. If it can be shown that a trait $A$ systematically causes higher fitness than trait $B$, we can, ceteris paribus, predict that trait $A$ will replace trait $B$ in the population. An explanation for the prevalence of trait $A$ can then be obtained through theoretical arguments or empirical data showing that individuals with trait $A$ systematically tend to have higher fitness than individuals with trait $B$. More generally, adaptive landscapes or fitness landscapes, which models fitness as a function of genotype frequencies (Wrightian landscapes) or phenotypes (Simpsonian landscapes) are useful explanatory devices (Reiss 2007; Svensson \& Calsbeek 2012; Svensson 2016). Some common approaches to studying selection are: 1) Selection-gradient analysis in which a measure of relative fitness is regressed against trait values to determine the direction and pattern of selection (Lande \& Arnold 1983; Arnold \& Wade 1984). This approach is particularly powerful in that regression on multiple traits can be used to distinguish direct selection on the trait itself from indirect selection stemming from correlation with other traits. 2) Causal manipulations in which traits are experimentally modified and resulting effects on a fitness measure are scored. For example, the selective effects of pollinators can be studied by comparing selection gradients between plants that are naturally pollinated with plants that are hand pollinated. More generally, the causal 
influence of some factor can be studied by comparing selection gradients with and without the factor present (Wade \& Kalisz 1990). 3) Optimality models in which design arguments are used to derive states of maximal fitness that can then be tested against observed trait values (Mitchell \& Valone 1990; Reeve \& Sherman 1993; Orzack \& Sober 2001). In cases of frequency-dependent selection the optimality approach may take the form of game-theoretical models in which optimal states are replaced with evolutionary stable strategies (ESS) or characterizations based on invadability of strategies. The term invasion fitness is sometimes used to characterize the fitness of a type when rare in a population. If invasion fitness exceeds mean fitness, the type may invade.

Arnold, S. J. and M. J. Wade. 1984. On the measurement of natural and sexual selection: theory. Evolution 38: 709-719.

Good explanation of selection-gradient analysis making clear how it applies to episodes of selection, and how it separates the study of selection from that of genetics and transmission. There is a companion paper with applications.

Lande, R. and S. J. Arnold 1983. The measurement of selection on correlated characters. Evolution 37:1210-1226.

This foundational paper introduced selection-gradient analysis, which rapidly became the chief tool for the empirical study of selection in nature. The selection gradient is best defined as a vector of derivatives of relative fitness on a set of traits, and this paper shows how the selection gradient can be obtained from a multiple regression of relative fitness on the traits.

Mitchell, W. A. and T. J. Valone 1990. The optimization research program: studying adaptations by their function. Quart. Rev. Biol. 65: 43-52.

This paper discusses the use of fitness optimization in the study of adaptation as a scientific research program in the sense of Lakatos. Identifies the core assumptions of the research program, which include the idea that fitness is optimized and that transmission effects can be ignored.

Orzack, S. H. and E. Sober (Eds.). 2001. Adaptationism and optimality, Cambridge University Press.

This edited volume contains many good and critical discussions of optimality models in the study of adaptation.

Reeve, H. K. and P. W. Sherman 1993. Adaptation and the goals of evolutionary research. Quart. Rev. Biol. 68: 1-32.

Influential review and argument about the meaning of adaptation. Argues for viewing adaptation as fitness optimization within defined constraints.

Reiss, J. O. 2007. Relative fitness, teleology, and the adaptive landscape. Evol. Biol. 34: 4-27. 
Discussion of the uses of the fitness concept in the adaptive landscape metaphor.

Svensson, E. I. 2016. Adaptive landscapes. In Encyclopedia of evolutionary biology. R. M. Kliman, Oxford: Academic Press: 9-15.

Non-technical review of uses of the adaptive landscape in current research.

Svensson, E. I. and R. E. Calsbeek (Eds.). 2012. The adaptive landscape in evolutionary biology, Oxford University Press.

This edited volume contains discussions of the history and different meanings of the concept of an adaptive landscape, as well as discussions of its use and ramifications in contemporary evolutionary biology.

Wade, M. J. and S. Kalisz 1990. The causes of natural selection. Evolution 44: 1947-1955.

Clear discussion of how to use selection-gradient analysis as a tool to test for causes of natural selection. Although the selection gradient is merely a description of a pattern of selection, comparison of selection gradients across environments or experimental treatments can test for causal mechanisms.

\section{Malthusian Fitness}

An episode of selection can be anything from a short event to a generation or more. Selection in continuous time can be described with differential equations by considering an infinitesimally short episode of selection. Here fitness differences become infinitesimally small and are better expressed as rates of change in numbers. If $d N_{i}=N_{i}^{\prime}-N_{i}$ is the change in number of type $i$ over a time interval $d t$, then the fitness of $i$ over this interval can be expressed as the Malthusian growth rate, $m_{i}=\left(W_{i}\right.$ - 1)/dt, so that $d N_{i}=W_{i} N_{i}-N_{\mathrm{i}}=m_{i} d t N_{i}$, which yields the standard differential equation for exponential growth:

$$
\frac{d N_{i}}{d t}=m_{i} N_{i}
$$

The rate of change of the total population size, $N$, is obtained by summing over types as $d N=\bar{m} N d t$, where $\bar{m}$ is mean fitness. The rate of change in frequency, $p_{i}$, of type $i$ is then obtained by calculation:

$$
\frac{d p_{i}}{d t}=\left(m_{i}-\bar{m}\right) p_{i}
$$

so that the relative fitness becomes the Malthusian growth rate of the type minus the mean Malthusian growth rate of the population. The term Malthusian fitness is used for fitness expressed as growth rate in continuous time, while the term Wrightian fitness is used for fitness as change in numbers in discrete time (Crow \& Kimura 1970, op. cit.). The two may be related as $\operatorname{Ln}\left[W_{i}\right]=m_{i} t$, where $t$ is the length of time selection is acting over. Wrightian fitnesses are relativized by division with the mean fitness, while Malthusian fitnesses are relativized by subtraction of mean fitness. The 
designation of fitness as population growth rate does not entail an assumption of exponential growth. The Malthusian fitness, as well as the Wrightian fitness, need not be constants, but are generally functions of population or environmental variables. For example, density-dependent selection occurs when relative fitnesses are functions of population density, and frequency-dependent selection occurs when relative fitnesses are functions of type frequencies. There are versions of the fundamental theorem and the Price theorem for continuous time based on Malthusian fitness (Price 1972b).

Price, G. R. 1972b. Extension of covariance selection mathematics. J. Hum. Genet., Lond. 35: 485-490.

Presents some extensions and variations of the Price theorem including to continuous time and to group selection.

\section{Measuring Fitness I: Fitness Components}

Fitness is a theoretical concept, but for empirical studies it has to be based on operational measurements in the form of statistics that represent the underlying theoretical entity. Common measurements are frequencies of survival or numbers of offspring (seeds, eggs, etc.). It is usually impractical to obtain measures of fitness that cover the entire life history of an organism, and most studies use proxy variables called fitness components, life-history traits that can be assumed to be positively related to fitness when all other factors are kept constant. The trick is to find a fitness component that adequately represents the causal influence of the selective factor under investigation. In a study of sexual selection on peacock tails, for example, the number of matings a male obtains may capture selection due to female preference even if it is not an adequate measure of the total selection acting on the male throughout its life. It is however important to consider the possibility of trade-offs between fitness components (Stearns 1992; Roff 1992). Trade-offs between components such as survival and reproduction, or size and number of offspring are inevitable due to inherent limitations in the time and resources available to an organism (Charnov 1993, 1997). This does not necessarily manifest in negative correlations between fitness components within populations however, because individuals may vary in how much resources they acquire as well as in how these are allocated among components (Van Nordwijk \& de Jong 1986; Houle 1991).

Charnov, E. L. 1993. Life history invariants: some explorations of symmetry in evolutionary ecology. Oxford University Press.

Elegant treatment of trade-offs between life-history traits derived from inherent symmetries in life-history theory.

Charnov, E. L. 1997. Trade-off-invariant rules for evolutionary stable life histories. Nature 387: 393-394.

Shows that fitness in a stable population (i.e. net reproductive output) can be generally written as a product of 1) survival to first breeding, 2) average rate of offspring production and 3) adult life span. This implies necessary tradeoffs between these components when selection reaches equilibrium. 
Houle, D. 1991. Genetic covariance of fitness correlates: what genetic correlations are made of and why it matters. Evolution 45: 630-648.

A population-genetics treatment of the acquistion-allocation model of Van Nordwijk \& de Jong (1986).

Roff, D. A. 1992. The evolution of life histories: theory and analysis. Chapman \& Hall.

Comprehensive review of life-history theory with emphasis on empirical research.

Stearns, S. C. 1992. The evolution of life histories. Oxford University Press.

Consise review of life-history theory.

Van Noordwijk, A. J. and G. De Jong 1986. Acquisition and allocation of resources: their influence on variation in life history tactics. Am. nat. 128: 137-142.

Shows that both positive and negative correlations between fitness components can result as a function of the relative amount of variation in acquistion and allocation of resources.

\section{Measuring Fitness II: Scaling and Transformation}

In measuring fitness it is important to respect its scale type. Fitness can not be arbitrarily transformed and still fulfill its theoretical role (Houle et al. 2011). Wagner (2010) provides a measurement-theoretical perspective on fitness and argues that Wrightian (discrete-event) fitness is on a ratio scale type while Malthusian (continuous-time) fitness is on an interval scale type. This means that the former allows only multiplication with a constant and must be relativized by division with the mean, while the latter allows translation and must be relativized by subtraction of the mean. These rules are often violated with resulting erroneous conclusions about selection. For example, Wagner (2010) showed that fitness epistasis and genotype-byenvironment interactions need be measured as deviations from a multiplicative scale for Wrightian fitness and on an arithmetic scale for Malthusian fitness. Wagner (2010), Chevin (2011) and Houle et al. (2011) cite examples in which erroneous conclusions have been drawn because researchers have confused these scales and for example measured genotype-by-environment interactions on Malthusian fitness as deviations from a multiplicative scale. Relative fitness does not allow any form of scaling or transformation at all. For example, using fitnesses relative to a maximum fitness can not substitute for relative fitness as predictors of frequency change and using alternative "relative" fitnesses will give quantitatively wrong results in both theoretical and empirical studies. Log absolute fitness may however be used as an approximation to (Wrightian) relative fitness.

Chevin, L.-M. 2011. On measuring selection in experimental evolution. Biol. Lett. 7: 210213.

Points out that Malthusian fitnesses are often erroneously compared on a multiplicative scale in studies of experimental evolution. 
Houle, D., C. Pélabon, G. P. Wagner and T. F. Hansen 2011. Measurement and meaning in biology. Quart. Rev. Biol. 86: 3-34.

General review of measurement-theoretical problems in biology. Discusses several examples of mismeasurement of selection and fitness in the literature.

Wagner, G. P. 2010. The measurement theory of fitness. Evolution 64: 1358-1376.

The first paper to do a measurement-theoretical analysis of fitness. Derives Wright's selection equations from utility theory based on assumptions about pairwise comparison of competitive ability. From this scaling properties and characterizations of epistasis and genotype-by-environment interaction in fitness are derived. Violations of these principles in experimental work are criticized.

\section{Fitness in Varying Environments}

Selection may not act uniformly on a whole population at once. Instead, the total selection experienced by a population throughout a generation (or across generations) is composed of many local episodes of selection at different spatio-temporal locations. The combined effect of such episodes can often be found by two simple rules for combining fitnesses. The first rule is that fitnesses pertaining to separate, parallel episodes of selection are combined as weighted arithmetic averages. If $W_{i}$ denotes the absolute fitness of selection in the i'th subpopulation, then selection in the total population can be described by using as fitness: $W=\Sigma_{i} p_{i} W_{i}$, where $p_{i}$ is the proportion of the population that belongs to subpopulation $i$ before selection. The second rule is that the (absolute or relative) fitnesses of sequential episodes of selection are combined multiplicatively. If $W_{t}$ denotes fitness at time $t$, then the combined fitness describing selection over subsequent non-overlapping time intervals is $W=\Pi_{t} W_{t}$. The last rule is often inaccurately presented by saying that one should use the geometric mean fitness (i.e. $\sqrt[T]{{ }_{t} W_{t}}$, where $T$ is the number of episodes). The geometric mean may be regarded as a scaling to compare with the fitness of a single episode (or generation), but it is the product and not the geometric mean that gives the correct combined fitness needed to predict changes in numbers or frequency. Derivations, discussions and extentions of these rules can be found in Dempster (1955), Levins (1962, 1968), Frank and Slatkin (1990), Frank (2011) and Engen and Sæther (2014). Hereford (2009), Bell (2010), Morrissey and Hadfield (2012), and Siepielski et al. (2013) are recent reviews and meta-analyses of spatio-temporal variation in selection.

Bell, G. 2010. Fluctuating selection: the perpetual renewal of adaptation in variable environments. Phil. Trans. R. Soc. B 365: 87-97.

Arguing that the traditional view of selection as a weak force is mistaken, and that large and fluctuating fitness differences are common in nature.

Dempster, E. R. 1955. Maintenance of genetic heterogeneity. Cold Spring Harbor Symp. Quant. Biol. 20: 25-32. 
May have been the first to derive the multiplication rule for fitness in a temporally-changing environment.

Engen, S. and B.-E. Sæther. 2014. Evolution in fluctuating environments: Decomposing selection into additive components of the Robertson-Price equation. Evolution 68: 854-865.

Generalizes the Price equation to include both environmental and demographic stochasticity in the same framework.

Frank S. A. 2011. Natural selection I. Variable environments and uncertain returns on investment. J. Evol. Biol. 24: 2299-2309.

The first in a series of seven papers presenting a general theory of natural selection. This paper focuses on effects of various types of variation in fitness.

Frank, S. A. and M. Slatkin 1990. Evolution in a variable environment. Am. nat. 136: 244260.

Reviews models of spatial and temporal fluctuations in selection and fitness. Develops a general framework for assessing the effects of different modes of variation in fitness.

Hereford, J. 2009. A quantitative survey of local adaptation and fitness trade-offs. Am. Nat. 173: 579-588.

A review of reciprocal-transplant experiments to test for local adaptation and fitness tradeoffs. Finds that local adaptation is common in the sense that most populations have higher fitness in their local environment.

Levins, R. 1962. Theory of fitness in a heterogeneous environment, I. The fitness set and adaptive function. Am. Nat. 96: 361-373.

Introduces the idea of a fitness set, which plots a parameterized curve as a function of fitness in different environments. The shape of this curve allows inferences about when selection favors specialized or generalist phenotypes.

Levins, R. 1968. Evolution in changing environments. Princeton University Press.

Classic work on the theory of fitness in heterogeneous environments.

Morrissey, M. B. and J. D. Hadfield. 2012. Directional selection in temporally replicated studies is remarkably consistent. Evolution 66: 435-442.

Important methodological paper assessing the influence of estimation error in inferences about temporal variation in selection gradients. Reevaluates a metaanalysis to conclude there is less temporal variation than previously thought.

Siepielski, A. M., K. M. Gotanda, M. B. Morrissey, S. E. Diamond, J. D. DiBattista and S. M. Carlson 2013. The spatial patterns of directional phenotypic selection. Ecol. Lett. 
16: $1382-1392$.

Meta-analysis of spatial variation in selection gradients.

\section{Fitness in Structured Populations}

A population may be structured into different age, sex or life-stage categories on which the patterns, factors and consequences of selection may be different. For example, the effect of selection tends to decrease with the age in the sense that total fitness is usually less sensitive to old-age fitness components than to young-age fitness components (e.g. Hamilton 1966). In general, the contribution of individuals of type $i$ and age (or stage) $x$ to the gamete pool at time $t$ will be $l_{x i} b_{x i} N_{i}\left(t-t_{x}\right)$, where $l_{x i}$ is the probability of surviving to age $x, b_{x i}$ is the fertility at age $x$, and $N_{i}\left(t-t_{x}\right)$ is the number of type $i$ at the zygote stage counting back the time $t_{x}$ separating stage $x$ from the zygote stage. Hence, the absolute fitness of type $i$ describing selection over a time interval $T$ is

$$
W_{i}=\frac{N_{i}(t)}{N_{i}(t-T)}=\Sigma_{x} Q_{x i} l_{x i} b_{x i}
$$

where $Q_{x i}=N_{i}\left(t-t_{x}\right) / N_{i}(t-T)$ accounts for different initial sizes of the age cohorts. If the type has a stable age structure then $Q_{x i}=\lambda_{\square}{ }^{T-t x}$, where $\lambda_{\square}$ is the growth rate of $i$ measured as the largest eigenvalue of the associated population projection matrix or Leslie Matrix (see Caswell 1989). The relative fitness is

$$
w_{i}=\frac{p_{i}(t)}{p_{i}(t-T)}=\frac{W_{i}}{\mathrm{E}_{t-T}\left[W_{i}\right]}
$$

where $\mathrm{E}_{t-T}\left[W_{i}\right]$ is the expected (mean) fitness of zygotes at time $t-T$. This setup is loosely based on Abugov (1988), who gives general equations for age- and sexstructured populations. With a lot of additional assumptions including stable age (or stage) structure and weak selection, the growth rate $\lambda$ can be used as a measure of fitness (Lande 1982). In continuous time similar assumptions give the Malthusian growth rate, $m$, obtained from the Euler equation,

$$
\int_{x} \mathrm{e}^{-m t} l_{x i} b_{x i}=1,
$$

as a measure of Malthusian fitness (Fisher 1930 op. cit.; Charlesworth 1994). In a population of constant size under weak selection one can set $Q_{x i} \approx 1$, and use the lifetime reproductive success, $W_{i}=\Sigma_{x} l_{x i} b_{x i}$, as a measure of fitness. This is easy to score by counting the total number of offspring from an individual and is thus commonly used in field studies of selection (e.g. Clutton-Brock 1988). Brommer (2000) reviews fitness concepts in life-history theory more generally.

Abugov, R. 1988. A Sex-specific quantitative genetic theory for life history and development. J. Theor. Biol. 132: 437-447.

One of several papers by Abugov developing general equations for describing selection in age- and sex-structured populations. 
Brommer, J. E. 2000. The evolution of fitness in life-history theory. Biol. Rev. 75: 377-404.

Reviews and compares fitness concepts used in life-history theory.

Caswell, H. 1989. Matrix population models: construction, analysis, and interpretation. Sinauer.

A clear and concise introduction to age- and stage-structured population models with material on models of selection. Also available in an expanded second edition.

Charlesworth, B. 1994. Evolution in age-structured populations. Sec ed. Cambridge University press.

A classic text on the population genetics of age-structured populations. The first edition came in 1980 .

Clutton-Brock, T. H. (Ed.) 1988. Reproductive success: Studies of individual variation contrasting breeding systems. Chicago University Press.

This edited volume reviews methods and field studies of reproductive success.

Hamilton, W. D. 1966. The moulding of senescence by natural selection. J. Theor. Biol. 12: $12-45$.

Classic paper developing formal theory of selection in age-structured populations. Shows that the sensitivity of fitness to fertility at age $x$ is proportional to the fraction of individuals alive at age $x$, and that the sensitivity of fitness to survival is proportional to the fraction of individuals alive multiplied with the reproductive value (expected contribution to future population growth) of individuals of age $x$.

Lande, R. 1982. A quantitative genetic theory of life history evolution. Ecology 63: 607-615.

A quantitative-genetics formulation of evolution in age-structured populations. Shows that the leading eigenvalue of the Leslie matrix can be used as a fitness measure under certain assumptions.

\section{Fitness and Levels of Selection}

Selection can take place on different hierarchical levels including the gene, the individual, groups or colonies of individuals, and whole species. The Price theorem can be used to decompose selection into different levels by treating selection at lower levels as transmission effects (Price 1972b op.cit.; Okasha 2006 op.cit; Gardner 2015). For example, the joint effects of group and individual selection can be described as

$$
\Delta \mathrm{E}[z]=\operatorname{Cov}\left[w_{g}\left(z_{g}\right), z_{g}\right]+\mathrm{E}_{g}\left[\operatorname{Cov}\left[w_{i}\left(z_{i}\right), z_{i}\right]+\mathrm{E}\left[w_{i}\left(z_{i}\right) \Delta z_{i}\right]\right],
$$


where $w_{g}$ is the relative fitness of a group calculated as the mean of the absolute fitnesses within the group divided by the mean of this across groups, $z g$ is the mean trait value of the group, and $w_{i}$ and $z_{i}$ the relative fitnesses and trait values of types within the groups. The $\mathrm{E}_{\mathrm{g}}$ denotes expectation over groups. According to this formalism selection occurs at a given level when the associated covariance term is not zero. It is always possible, however, to describe selection in terms of lower-level entities by calculating marginal fitnesses. For example, group-level fitness can be reduced to individual-level fitness by averaging the fitness of individuals of a type taking account of what groups they occur in, and individual-level fitness can be reduced to gene-level fitness by taking the average fitness of the individuals in which a gene (i.e. allele) occurs. Sober and Wilson (1998; Wilson 1980; Sober 1984 op. cit.) argued that group selection occurs when there is a causal interaction between the group and the environment; i.e. when the group fitness is causally determined by the composition of the group. The overwhelming focus on individual-level selection in evolutionary biology may be a consequence of integrated individuals doing most of the causal interaction with the environment. The evolution of "individuality" in transitions to multicellularity or coloniality may then be associated with shifts in the main level of selection. Arguments for the primacy of the gene as in Williams (1966) and Dawkins (1976) focus instead on the stability of the gene as a replicator. This ignores the distinction between selection and transmission, and is perhaps better phrased as an argument for the gene as the unit of evolution than as the unit of selection. Finally, selection may happen at the level of species through differential rates of speciation and extinction (Jablonski 2008; Chevin 2016).

Chevin, L.-M. 2016. Species selection and random drift in macroevolution. Evolution 70: 513-525.

Recent theoretical paper describing species selection (cladogenesis) and individual selection (anagenesis) in common framework.

Dawkins, R. 1976. The selfish gene. Paladin.

A classic brilliantly-written argument for a gene-level perspective on natural selection and adaptation.

Gardner, A. 2015. The genetical theory of multilevel selection. J. Evol. Biol. 28: 305-319.

Recent formulation of multilevel selection based on decomposition of the Price equation. Handles within-group population structure.

Jablonski, D. 2008. Species selection: Theory and data. Ann. Rev. Ecol. Evol. Syst. 39: 501524.

General review of species selection.

Sober, E. and D. S. Wilson. 1998. Unto others: The evolution and psychology of unselfish behavior. Harvard University Press.

Book on the evolution and psychology of altruism with much material on the levels of selection. Argues that group-level adaptations, i.e. traits evolved due to group 
selection, are common, and particularly in humans.

Williams, G. C. 1966. Adaptation and natural selection: a critique of some current evolutionary thought. Princeton University Press.

The classic critique of group selection and best-for-the-species-style arguments in evolution. This book rendered group selection a near taboo for decades.

Wilson, D. S. 1980. The natural selection of populations and communities. Benjamin Cummings.

Defends the importance of group selection in evolution. Emphasis on "trait groups", which are possibly ephemeral collections of individuals interacting as a group. Shows that selection among such groups may be a powerful evolutionary force.

\section{Inclusive Fitness}

Alleles that make individuals behave altruistically towards other individuals that are likely to carry the same allele may be favored by selection even when they reduce the individual-level fitness of the altruist. Hamilton (1963) introduced the concept of inclusive fitness to describe this phenomenon on the individual level. The inclusive fitness of an altruist is

$$
W=r B-C,
$$

where $B$ is the benefit to the recipient of the altruistic act, $C$ is the cost to the altruist, and $r$ is the coefficient of relatedness between the two (i.e. the probability that an allele in the altruist has an allele identical by descent in the recipient). From this follows Hamilton's rule that altruism evolves (by kin selection) when $r B>C$. Inclusive fitness may also be understood beyond kin selection if $r$ is interpreted as a correlation between the breeding values of the actor and recipient regardless of whether they are relatives (e.g. Grafen 1985; Queller 1992). Whether the evolution of altruism and related traits is best understood as group selection, kin selection or gene selection is a topic of continuing debate. Frank $(1998,2013)$ reviews these topics.

Frank, S.A. 1998. Foundations of social evolution, Princeton university press.

General review of the theoretical basis for social evolution. Much material on natural selection and the fitness concept. Covers the Price equation and kin selection in detail.

Frank, S.A. 2013. Natural selection. VII. History and interpretation of kin selection theory. $J$. Evol. Biol. 28: 1151-1184.

Review of the history and interpretation of kin-selection theory.

Grafen, A. 1985. A geometric view of relatedness. Oxford surveys in evolutionary biology 2: 28-89.

Discusses and generalizes the interpretation of the relatedness coefficient, $r$, in 
Hamilton's rule. Derives the rule from the Price theorem.

Hamilton, W. D. 1963. The evolution of the altruistic behavior. Am. Nat. 98: 353-356.

First of a series of papers by Hamilton introducing inclusive fitness and what has become known as Hamilton's rule for analyzing social evolution.

Queller, D. C. 1992. Quantitative genetics, inclusive fitness, and group selection. Am. Nat. 139: 540-558.

Develops a quantitative-genetics formulation of kin selection and inclusive fitness.

\section{Fitness in Finite Populations}

To characterize fitness in a finite population we need to distinguish changes in frequencies of types due to selection from those due to genetic drift (i.e. random sampling). With a finite number of individuals the fitness of a type $i$, defined as $W_{i}=$ $N_{i}^{\prime} / N_{i}$, becomes a random variable (Hansen 2017). It is natural to take the expectation of this as a measure of underlying fitness and the variance as a measure of genetic drift. Drift and selection may interact, however. Because the relative fitness of a type is a concave function of its absolute fitness, variation in realized absolute fitness will reduce the (expectation of) relative fitness (Gillespie 1977; Sober 2001; Orr 2007). Hence, types that exhibit less variance in the number of offspring they produce will be favored by selection in small populations. Even in large populations genetic drift is important for the selection dynamics of rare types. For example, a new mutation existing as a single copy will usually be lost by drift even if it has a selective advantage. To a first approximation, the fixation probability of a single advantageous mutation with a Poisson offspring distribution in a large stable population is $2 s$, with $s$ $=w-1$, where $w$ is the relative fitness of the mutation (Haldane 1927; Bürger \& Ewens 1995; see also Otto \& Whitlock 1997). Hence, mutations with an $s=1 \%$ selective advantage are lost $98 \%$ of the time.

Bürger, R. and W. Ewens. 1995. Fixation probabilities of additive alleles in diploid populations. J. Math. Biol. 33: 557-575.

Gives an accurate approximation for the probability of fixation of an advantageous allele in a finite population.

Gillespie, J. H. 1977. Natural selection for variance in offspring numbers: a new evolutionary principle. Am. Nat. 111: 1010-1014.

One of several papers by Gillespie exploring the consequences of variation in number of offspring for selection dynamics in finite populations.

Haldane, J. B. S. 1927. A mathematical theory of natural and artifical selection. V. selection and mutation. Proc. Camb. Phil. Soc. 23: 838-844.

First derivation of the fixation probability of a single new advantageous mutation as $2 s$ in a population of constant size with a Poisson offspring distribution. 
Hansen, T. F. 2017. On the definition and measurement of fitness in finite populations. $J$. Theor. Biol. 419: 36-43.

A discussion of the meaning of fitness in finite populations. Gives general equations for the mean and variance of relative fitness in the presence of demographic stochasticity, and criticizes some inaccuracies in the literature.

Orr, H. A. 2007. Absolute fitness, relative fitness, and utility. Evolution 61: 2997-3000.

A discussion of why selection is "risk averse" in that it tends to favor types with less variance in absolute fitness.

Otto, S. P. and M. C. Whitlock. 1997. The probability of fixation in populations of changing size. Genetics 146: 723-733.

Shows that the probability of fixation of a new advantageous mutation in a population with Malthusian growth rate $m$ is approximately $2(s+m)$. Hence, fixation of an advantageous mutation is more probable in a growing population.

Sober, E. 2001. The two faces of fitness. In Singh, R. S., C. B. Krimbas, D. B. Paul \& J. Beatty (Eds.). Thinking about evolution: Historical, Philosophical, and political perspectives. Cambridge University Press. Pp. 309-321.

A discussion of the fitness concept. Focus on issues having to do with time frame and variation in offspring number.

\section{Fitness in the Philosophy of Biology}

The concept of fitness has generated interest among philosophers of biology, and has amassed much commentary that will not be detailed here. Good entry points can be found in books by Sober (1984a op. cit., 1984b, 1993), Brandon (1990), Okasha (2006 Op.cit.), and Godfrey-Smith (2009 op. cit.). One focus of this literature has been to solve a perceived tautology problem. If fitness is defined as survival, then the survival of the fittest sounds circular, and many have charged that this makes explanations based on natural selection untestable. This includes Karl Popper's infamous reference to the theory of natural selection as a metaphysical research program. Popper later changed his mind on the testability and blamed sloppy definitions by biologists such as Waddington, Fisher, Haldane and Simpson for generating the misunderstanding that natural selection was tautological (Popper 1978; Elgin \& Sober 2017). A concept like fitness does not get its meaning from definitions however, but from its theoretical context. As is obvious to practicing evolutionary biologists, the theory is not tautological because hypotheses about casual links between traits and fitness are testable. A step towards formally solving the tautology problem was the propensity interpretation of fitness (Brandon 1978; Mills \& Beatty 1979). Here it was argued that fitness needs to be seen as a disposition and not as actual reproductive success. If one of two identical twins standing next to each other is killed by lightning, while the other goes on to reproduce, they still have the same fitness, because they the had the same properties and the same propensity to 
reproduce. The propensity interpretation has been a focus for continuing debate (e.g. Beatty \& Finsen 1989; Byerly \& Michod 1991). Other influential ideas have focused on the difficulty (impossibility) to link fitness to well-defined biological properties. It has been suggested that fitness should be treated as a primitive, an axiomatic entity that can not be defined from within the theory (Williams 1970 op. cit.), or as a supervenient property that can not be reduced to any specific biological properties (Rosenberg 1978; Sober 1984 op. cit.). Philosophers of biology have also taken interest in the levels-of-selection debates in the light of theory reduction (e.g. Okasha 2006 op. cit.).

Beatty, J. and S. Finsen. 1989. Rethinking the propensity interpretation: A peek inside Pandora's box. In Ruse, M. (Ed.). What the philosophy of biology is. Pp. 17-30.

A critical reexamination of the propensity interpretation.

Brandon, R. N. 1978. Adaptation and evolutionary theory. Studies in the history and philosophy of science 9: 181-206.

Discusses the concept of "adaptedness", and argues that it should be seen a dispositional concept.

Brandon, R. N. 1990. Adaptation and environment. Princeton University Press.

Philosophical treatment of adaptation and selection. Discusses teleology, levels of selection, and the concept of environment. Uses the concept of screening off as a tool to recognize the causal level of selection. If the fitness of an individual is determined by its phenotype regardless of its genotype, then the phenotype screens off the genotype and must be considered the causal agent in selection.

Byerly, H. C. and R. E. Michod. 1991. Fitness and evolutionary explanation. Biology and Philosophy 6: 1-22.

Broad criticism of different fitness concepts, including the propensity interpretation, with many comments in the same issue.

Elgin, M. and E. Sober. 2017. Popper's shifting appraisal of evolutionary theory. Hopos 7: 31-55.

A broad analysis of Popper's shifting (or not) positions on natural selection.

Mills, S. and J. Beatty. 1979. The propensity interpretation of fitness. Philosophy of science 46: 263-286.

Influential paper presenting the propensity interpretation of fitness. Argues that the definitions of fitness given in the biological literature are not reflecting the use of fitness in biological explanation, and invites the charge of circularity. This is solved by recognizing fitness as a dispositional concept, the ability to survive and reproduce rather than the actual realization of such. 
Popper, K. 1978. Natural selection and the emergence of mind. Dialectica 32: 339-355.

Paper given as the first Darwin lecture at Darwin College in Cambridge. Discusses the scientific status of natural selection and retracts his earlier characterization of natural selection as tautological. Popper's characterization of the theory of natural selection as a "metaphysical research program" was in any case not intended to be derogatory ("research program" would have been more accurate), and he used it to show how a useful theory could have tautological elements. Discusses the evolution of mind.

Rosenberg, A. 1978. The supervenience of biological concepts. Philosophy of science 45: 368-386.

Introduces the idea of fitness as supervenient on biological properties. This means that although fitness is determined by biology, it is not defined by biological properties and can be realized in many different ways. Uses this to analyze some misunderstandings of selection.

Sober, E. (Ed.) 1984b. Conceptual issues in evolutionary biology: An anthology. Bradford books.

An anthology of papers in the philosophy of biology. Many classic papers on fitness and levels of selection. There are also second and third editions with less material on fitness and selection.

Sober, E. 1993. Philosophy of biology. Westview press.

Introduction to the philosophy of evolutionary theory with a chapter on fitness.

\section{Alternative Characterizations of Fitness}

Fitness has been related to, and sometimes defined in terms of, many specific biological or physical properties including energy, entropy, information, biomass, survival, adaptation and optimality. For example, there is an old line of thought linking natural selection to energy maximization going back to Lotka (1925), and later being promoted by systems ecologists such as Odum and others. The idea is that natural selection maximizes the acquisition, control, or flow of energy (e.g. Van Valen 1976). Synonymizing fitness with energy is broadly inconsistent with its contemporary use in evolutionary biology however, and abundant counter examples to selection maximizing energy can be found in consideration of sexual selection and soft selection. Attempts at linking fitness to measures of information or entropy also regularly appear (Maynard Smith 1999). Kimura (1961) is a classic and Frank (2009, 2012c) a recent attempt at arguing that natural selection accumulates and maximizes information. Fitness is also regularly equated with adaptation and optimality. This ignores the separation between selection and transmission, and tends to obscure the precise role of fitness. Often this is just lax writing rendering fitness a synonym of adaptation. A formal characterization of fitness maximation has been developed by Grafen in an attempt at defending optimization as used in behavioral ecology and related fields (Grafen 2014). There are other characterizations of fitness based on 
alternative theoretical contexts. For example, there are situations in which it is necessary to analyze selection over more than one generation. Providing many offspring to the next generation is not going to help if they are all sterile. Hence, fitness is sometimes measured as number of grand offspring. There is nothing special about two generations however, and it has been suggested that fitness should be defined over many generations or as fixation probabilities, long-term growth rates or times to extinction (e.g. Thoday 1953; Cooper 1984; Metz et al. 1992). These approaches suffer from lack of generality in the assumptions they must make about transmission and ecology, from arbitrariness in the choice of time scale and from rarely being empirically operational (see e.g. Brandon 1990 op. cit.; Sober 2001 op. cit. for criticism). More general models of simple episodes of selection can always be used as elements in predicting long-term dynamics. The standard models are also not limited to counting offspring, but allow weighting with predicted survival or reproductive value. One big seed may give higher fitness than two small seeds.

Cooper, W. S. 1984. Expected time to extinction and the concept of fundamental fitness. $J$. Theor. Biol. 107: 603-629.

Argues that expected time to extinction should be used as the fundamental measure of fitness.

Frank, S. A. 2009. Natural selection maximizes Fisher information. J. Evol. Biol. 22: 231244.

Shows that natural selection increases a formal measure of information. Frank (2012c) extends this work.

Frank, S. A. 2012c. Natural selection. V. How to read the fundamental equations of evolutionary change in terms of information theory. J. Evol. Biol. 25: 2377-2396.

Derives some general expressions for the change of mean fitness as change in measures of information. Claims that selection can be described as increase in information, and reviews other work on links between selection and information.

Grafen, A. 2014. The formal darwinism project in outline. Biology \& Philosophy 29: 155174.

Grafen's formal Darwinism project is an attempt to formalize the theory of natural selection and fitness presented in a series of technical papers. This paper is a nontechnical review published together with a series of comments by biologists and philosophers ranging from the adulatory to the dismissive.

Kimura, M. 1961. Natural selection as the process of accumulating genetic information in adaptive evolution. Genet. Res. 2: 127-140.

Computes the information gain by fixation of an advantageous mutation by comparing it to the probability of fixing a random mutation. Uses this to compute the rate at which bits of information are accumulated in lineages.

Lotka, A. J. 1925. Elements of physical biology. Baltimore, Williams \& Wilkins. 
A grand attempt at identifying physics-style laws in biology. Suggests that natural selection acts to optimize energy flow.

Maynard Smith, J. 1999. The idea of information in biology. Quart. Rev. Biol. 74: 395-400.

A general review of attempts to use information theory in biology.

Metz, J. A. J., R. M. Nisbet and S. A. H. Geritz. 1992. How should we define 'fitness' for general ecological scenarios? TREE 7: 198-202.

Argues that fitness should be understood as a long-term growth rate; specifically as a Lyapunov exponent.

Thoday, J. 1953. Components of fitness. Symposium of the society for experimental biology 7: 96-113.

Suggests to view fitness as number of offspring in the long run, such as after 100 million years.

Van Valen, L. M. 1976. Energy and evolution. Evolutionary Theory 1: 179-229.

One of several papers by Van Valen arguing that natural selection acts to maximize energy, and suggesting to define fitness in terms of energy.

\section{Acknowledgements}

I thank David Houle and an anonymous reviewer for useful comments on an earlier version of the manuscript. 